\title{
The Role of hOGG1 C1245G Polymorphism in the Susceptibility to Lupus Nephritis and Modulation of the Plasma 8-OHdG in Patients with Systemic Lupus Erythematosus ${ }^{\dagger}$
}

\author{
Hui-Ting Lee ${ }^{1,2,3,4}$, Chen-Sung Lin ${ }^{1,2,5}$, Chyou-Shen Lee ${ }^{3,4,6}$, Chang-Youh Tsai ${ }^{1,2,7, *}$ and \\ Yau-Huei Wei ${ }^{1,2,3,8, *}$
}

1 Institute of Clinical Medicine, National Yang-Ming University, Taipei 112, Taiwan; E-Mails: htlee1228@gmail.com (H.-T.L.); doc2765c@ms59.hinet.net (C.-S.L.)

2 Faculty of Medicine, National Yang-Ming University, Taipei 112, Taiwan

3 Department of Medicine, Mackay Medical College, New Taipei City 252, Taiwan; E-Mail: amy@mmh.org.tw

4 Division of Allergy, Immunology and Rheumatology, Department of Internal Medicine, Mackay Memorial Hospital, Taipei 104, Taiwan

5 Division of Thoracic Surgery, Taipei Hospital, Ministry of Health and Welfare, New Taipei City 242, Taiwan

6 Mackay Junior College of Medicine, Nursing, and Management, New Taipei City 252, Taiwan

7 Division of Allergy, Immunology and Rheumatology, Department of Medicine, Taipei Veterans General Hospital, Taipei 112, Taiwan

8 Institute of Biochemistry and Molecular Biology, National Yang-Ming University, Taipei 112, Taiwan

$\dagger$ A portion of the data, the plasma levels of $8-\mathrm{OHdG}$, has been published in the scientific journal "Clinical \& Experimental Immunology (CEI)".

* Authors to whom correspondence should be addressed;

E-Mails: cytsai@vghtpe.gov.tw (C.-Y.T.); joeman@ym.edu.tw (Y.-H.W.);

Tel.: +886-2-2871-2121 (ext. 3366) (C.-Y.T.); +886-2-2826-7118 (Y.-H.W.);

Fax: +886-2-2826-4843 (Y.-H.W.).

Academic Editors: Chak-Sing Lau and Vera Sau-Fong Chan

Received: 26 November 2014 / Accepted: 29 January 2015 / Published: 9 February 2015

\footnotetext{
Abstract: We investigated whether the $\mathrm{C} 1245 \mathrm{G}$ polymorphism of human 8-oxoguanine glycosylase $1(h O G G 1)$ gene confers the susceptibility to systemic lupus erythematosus (SLE) occurrence of lupus nephritis and affects the plasma level of 8-hydroxy-2'-deoxyguanosine
} 
(8-OHdG) in patients with SLE. A total of 45 healthy controls and 85 SLE patients were recruited. The $\mathrm{C} 1245 \mathrm{G}$ polymorphism of the $h O G G 1$ gene was determined by direct sequencing. The frequency of occurrence of the hOGG1 $1245 \mathrm{GG}$ genotype in SLE patients was $31.8 \%(27 / 85)$, which is lower than that of healthy controls of $53.3 \%(24 / 45)$. Thirty-three (33/85, 38.8\%) SLE patients developed lupus nephritis. Significantly, SLE patients harboring the $h O G G 11245 \mathrm{GG}$ genotype had a higher incidence to develop lupus nephritis than did those harboring the hOGG1 $1245 \mathrm{CC}$ or CG genotype (15/27, 55.6\% $v s .18 / 58,31.0 \%, p=0.031$ ). Divided into subgroups, SLE patients harboring the $h O G G 1$ 1245 GG genotype had the highest plasma levels of 8-OHdG among patients with all genotypes, with regard to the coexistence of lupus nephritis ( $p=0.020$, ANOVA), including those with nephritis harboring the hOGG1 $1245 \mathrm{CC}$ or CG genotypes ( $p=0.037)$, those without nephritis harboring the hOGG1 1245 GG genotype $(p=0.050)$, and those without nephritis harboring the $h O G G 11245 \mathrm{CC}$ or CG genotype $(p=0.054)$. We conclude that the $\mathrm{C} 1245 \mathrm{G}$ polymorphism of $h O G G 1$ may be one of the factors that confer the susceptibility to lupus nephritis and modulate the plasma level of 8-OHdG in patients with SLE.

Keywords: 8-hydroxy-2'-deoxyguanosine (8-OHdG); human 8-oxoguanine glycosylase 1 ( $h O G G$ l) $\mathrm{C} 1245 \mathrm{G}$ polymorphism; lupus nephritis; systemic lupus erythematosus (SLE)

\section{Introduction}

Systemic lupus erythematosus (SLE) is a systemic autoimmune disease characterized by the generation of arrays of pathogenic auto-antibodies that cause cascades of organ damages [1]. Similar to several human degenerative diseases, elevated reactive oxygen species (ROS) and ROS-triggered oxidative damages have been demonstrated to be involved in the pathogenesis of SLE [2-5]. Theoretically, ROS can attack the intracellular molecules randomly, e.g., protein, lipid, RNA and DNA, and thereby cause cellular dysfunction. Among these oxidative DNA damages, the formation of 8-hydroxy-2'-deoxyguanosine (8-OHdG), an oxidative damage product of 2'-deoxyguanine (dG), is the most common one [6]. Normally, the $\mathrm{dG}$ is matched with 2'-deoxycytosine (dC) during DNA replication. However, if the 8-OHdG is not repaired efficiently and accumulated in the affected tissues, it would pair wrongly with 2'-deoxyadenine $(\mathrm{dA})$ rather than $\mathrm{dC}$, and cause a $\mathrm{G} \rightarrow \mathrm{C}$ to $\mathrm{T} \rightarrow \mathrm{A}$ transversion [7]. As a result, if the affected gene harbors $8-\mathrm{OHdG}$, functional impairment may subsequently develop.

In human cells, 8 -OHdG is mainly repaired by human 8-oxoguanine glycosylase 1 ( $h O G G l$ ) through the base excision repair mechanism [8]. There have been three hOGG1 genotypes identified because of a C to G shifting at base-pair (bp) 1245 (C1245G) in exon 7 of the hOGG1 gene. Such a shifting causes a serine (Ser) to cysteine (Cys) substitution at codon 326 during translation. This phenomenon is referred to as $h O G G 1 \mathrm{C} 1245 \mathrm{G}$ polymorphism. Considering the DNA repair efficiency, homozygous $h O G G 11245$ CC genotype has the highest DNA repair activity followed by the heterozygous $h O G G 11245 \mathrm{CG}$ genotype and then the homozygous $h O G G 11245$ GG genotype in order [9]. This discrepancy in $h O G G 1$ repair activity implies that $h O G G 11245$ GG genotype with lower efficiency in DNA repair might be related to a higher level of 8-OHdG in human tissues [10], 
and may contribute to the development of several human degenerative diseases, including type 2 diabetes mellitus [11], Huntington's disease [12], chronic obstructive pulmonary disease [13], Graves' ophthalmopathy [14], and higher susceptibility to cancer formation, including lung cancer [15] and esophageal squamous cell carcinoma [16]. However, the role of $h O G G 1 \mathrm{C} 1245 \mathrm{G}$ polymorphism in the pathogenesis of SLE has remained obscure.

Lupus nephritis is a serious clinical manifestation of SLE. Studies revealed that excessive ROS production and oxidative stress may exacerbate inflammation, dampen antioxidant capacity and lead to tissue damage and the formation of lupus nephritis in SLE patients [3,17]. Furthermore, some authors also reported that hOGG1 $1245 \mathrm{GG}$ genotype is related to higher 8-OHdG content in patients with end-stage renal disease (ESRD) $[18,19]$. In one of our previous studies, we demonstrated that SLE patients had higher plasma levels of 8-OHdG than did healthy controls [20]. In light of these findings, we aimed to appraise (1) whether the hOGG1 1245 GG genotype contributes to a higher rate of SLE development and higher plasma level of 8-OHdG; (2) whether the hOGG1 1245 GG genotype confers a higher incidence to the development of nephritis in SLE patients; and (3) whether the hOGG1 1245 GG genotype modulates the plasma level of 8-OHdG in SLE patients with or without nephritis.

\section{Results and Discussion}

\subsection{Distributions of hOGG1 C1245G Polymorphism in Healthy Controls and SLE Patients}

The genotypes of $h O G G 1 \mathrm{C} 1245 \mathrm{G}$ polymorphisms are illustrated in Figure 1 . As revealed by direct sequencing, among the 45 healthy controls, 3 (6.7\%) had the CC, 18 (40.0\%) had the CG and 24 (53.3\%) had the GG genotype, respectively. On the contrary, among the 85 SLE patients, 14 (16.5\%) had the CC genotype, 44 (51.8\%) had the CG genotype and 27 (31.8\%) had the GG genotype, respectively. For the subsequent comparative analysis, CC genotype plus CG genotype were gathered as one group in this study (Table 1).

As shown in Table 1, among the 85 SLE patients, 27 (31.8\%) harbored the GG genotype, and the rate was significantly lower than the $24(53.3 \%)$ of the 45 healthy controls $(p=0.017)$. For further comparisons, healthy controls harboring the CC or CG genotypes were designated as Group A, healthy controls harboring the GG genotype were designated as Group B, SLE patients harboring the CC or CG genotypes were designated as Group C and SLE patients harboring the GG genotype were designated as Group D, respectively.

\subsection{Levels of Plasma 8-OHdG in Healthy Controls and SLE Patients Based on the hOGG1 Gene Polymorphisms}

As we reported previously, the plasma $8-\mathrm{OHdG}$ levels $(\mathrm{M} \pm \mathrm{SD})$ of the 45 healthy controls and 85 SLE patients were $0.157 \pm 0.038$ and $0.225 \pm 0.082 \mathrm{ng} / \mathrm{mL}$, respectively [20]. SLE patients had significantly higher plasma $8-\mathrm{OHdG}$ levels as compared with healthy controls $(p<0.001)$, regardless of their hOGG1 gene polymorphism of CC/CG genotypes (Group C vs. Group A, $0.217 \pm 0.059$ vs. $0.161 \pm 0.036 \mathrm{ng} / \mathrm{mL}, p<0.001$ ) or GG genotype (Group D vs. Group B, $0.243 \pm 0.117$ vs. $0.155 \pm 0.041 \mathrm{ng} / \mathrm{mL}, p=0.001$ ) (Table 2). Those harboring hOGG1 1245 GG genotype did not have a higher level of plasma $8-\mathrm{OHdG}$ than those harboring $\mathrm{CC}$ or $\mathrm{CG}$ genotypes in healthy controls (Group B 
vs. Group A, $0.155 \pm 0.041$ vs. $0.161 \pm 0.036 \mathrm{ng} / \mathrm{mL}, p=0.614)$. A mild elevation of plasma 8-OHdG levels was observed in SLE patients with GG genotype (Group D vs. Group C, $0.243 \pm 0.117 v s$. $0.217 \pm 0.059 \mathrm{ng} / \mathrm{mL}, p=0.289$ ), although it was not obvious. Among all subjects, Group D (patients with the hOGG1 1245 GG genotypes) had the highest plasma levels of 8-OHdG ( $p<0.001$, ANOVA, Table 2 and Figure 2).
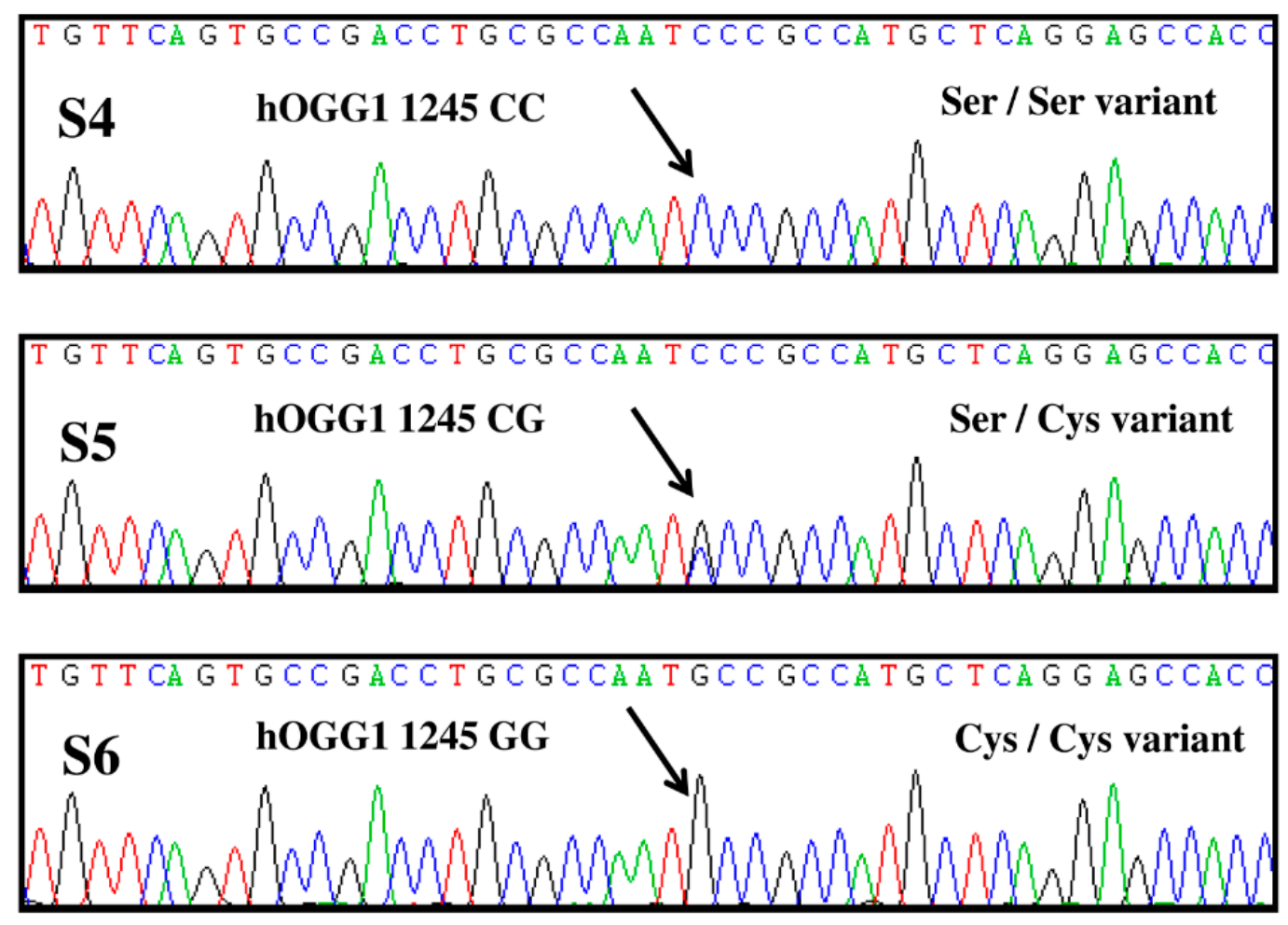

Figure 1. Illustrations are the results of direct sequencing of the DNA segment harboring the hOGG1 gene. Green, red, blue and black colors represent A, T, C and G, respectively. systemic lupus erythematosus (SLE) patient S4 harbored a single C peak at bp 1245 (homozygous $\mathrm{C} / \mathrm{C}$ allele) and belonged to the homozygous $\mathrm{CC}$ genotype. SLE patient S6 harbored a single peak of $\mathrm{G}$ at bp 1245 (homozygous $\mathrm{G} / \mathrm{G}$ allele) and belonged to the homozygous human 8-oxoguanine glycosylase 1 (hOGGl) GG genotype. SLE patient S5 harbored concurrent $\mathrm{C}$ and $\mathrm{G}$ peaks at bp1245 (heterozygous $\mathrm{C} / \mathrm{G}$ allele) and thus belonged to the heterozygous $\mathrm{CG}$ genotype.

Table 1. Distribution of $h O G G 1$ gene polymorphisms in healthy controls and SLE patients.

\begin{tabular}{cccc}
\hline & \multicolumn{2}{c}{$\boldsymbol{h O G G 1 \text { C1245G Polymorphisms }}$} & \\
\cline { 2 - 3 } Subjects (Case Number, \%) & $\begin{array}{c}\boldsymbol{h} \text { OGG1 1245 CC or } \\
\text { CG Genotype }\end{array}$ & $\begin{array}{c}\boldsymbol{h} \text { OGG1 GG } \\
\text { Genotype }\end{array}$ & \\
& $21(46.7)$-Value \\
\hline Healthy controls $(n=45,100)$ & $58(68.2)$-Group A & $24(53.3)$-Group B & $0.017 *$ \\
SLE patients $(n=85,100)$ & $27(31.8)$-Group D & \\
\hline
\end{tabular}

Group A: Healthy controls harboring the hOGG1 1245 CC or CG genotypes; Group B: Healthy controls harboring the hOGG1 1245 GG genotype; Group C: SLE patients harboring the hOGG1 $1245 \mathrm{CC}$ or CG genotype; Group D: SLE patients harboring the hOGG1 1245 GG genotype, * Comparison was made between healthy controls and SLE patients with regard to GG genotype. 
Table 2. Levels of plasma 8-hydroxy-2'-deoxyguanosine (8-OHdG) in healthy controls and SLE patients as related to the $h O G G 1$ gene polymorphisms.

\begin{tabular}{ccccc}
\hline & $\begin{array}{c}\text { Plasma 8-OhdG } \\
(\mathbf{M} \pm \mathbf{S D}, \mathbf{n g} / \mathbf{m L})\end{array}$ & & $\begin{array}{c}\text { Plasma 8-OHdG } \\
(\mathbf{M} \pm \mathbf{S D} \mathbf{n g} / \mathbf{m L})\end{array}$ & $\boldsymbol{p}$-Value \\
\hline $\begin{array}{c}\text { Healthy controls } \\
(n=45)\end{array}$ & $0.157 \pm 0.038$ & SLE patients $(n=85)$ & $0.225 \pm 0.082$ & $<0.001[20]$ \\
\hline $\begin{array}{c}h O G G 1 \text { C1245G } \\
\text { polymorphisms }\end{array}$ & $0.161 \pm 0.036$ & $\begin{array}{c}h O G G 1 \text { C1245G } \\
\text { polymorphisms }\end{array}$ & \\
\hline $\begin{array}{c}\text { Group C }(\mathrm{CC} \text { or CG } \\
\text { genotypes, } n=58)\end{array}$ & $0.217 \pm 0.059$ & $<0.001^{*}$ \\
\hline $\begin{array}{c}\text { Genotypes, } n=21) \\
\text { Group B }(\mathrm{GG} \\
\text { genotype, } n=24)\end{array}$ & $0.155 \pm 0.041$ & $\begin{array}{c}\text { Group D }(\mathrm{GG} \\
\text { genotype, } n=27)\end{array}$ & $0.243 \pm 0.117$ & $0.001^{*}$ \\
\hline$p$-value & $0.614^{* *}$ & $p$-value & $0.289^{* *}$ & $<0.001^{* * *}$ \\
\hline
\end{tabular}

M, mean, SD, standard deviation; * compared between healthy controls harboring the CC or CG genotypes (Group A) and SLE patients harboring the CC or CG genotypes (Group C), and between healthy controls harboring the GG genotype (Group B) and SLE patients harboring the GG genotype (Group D); ** compared between healthy controls harboring the GG genotype (Group B) and those harboring the CC or CG genotypes (Group A), or between SLE patients harboring the GG genotype (Group D) and those harboring CC or CG genotypes (Group C); *** compared among Groups A, B, C and D (ANOVA).

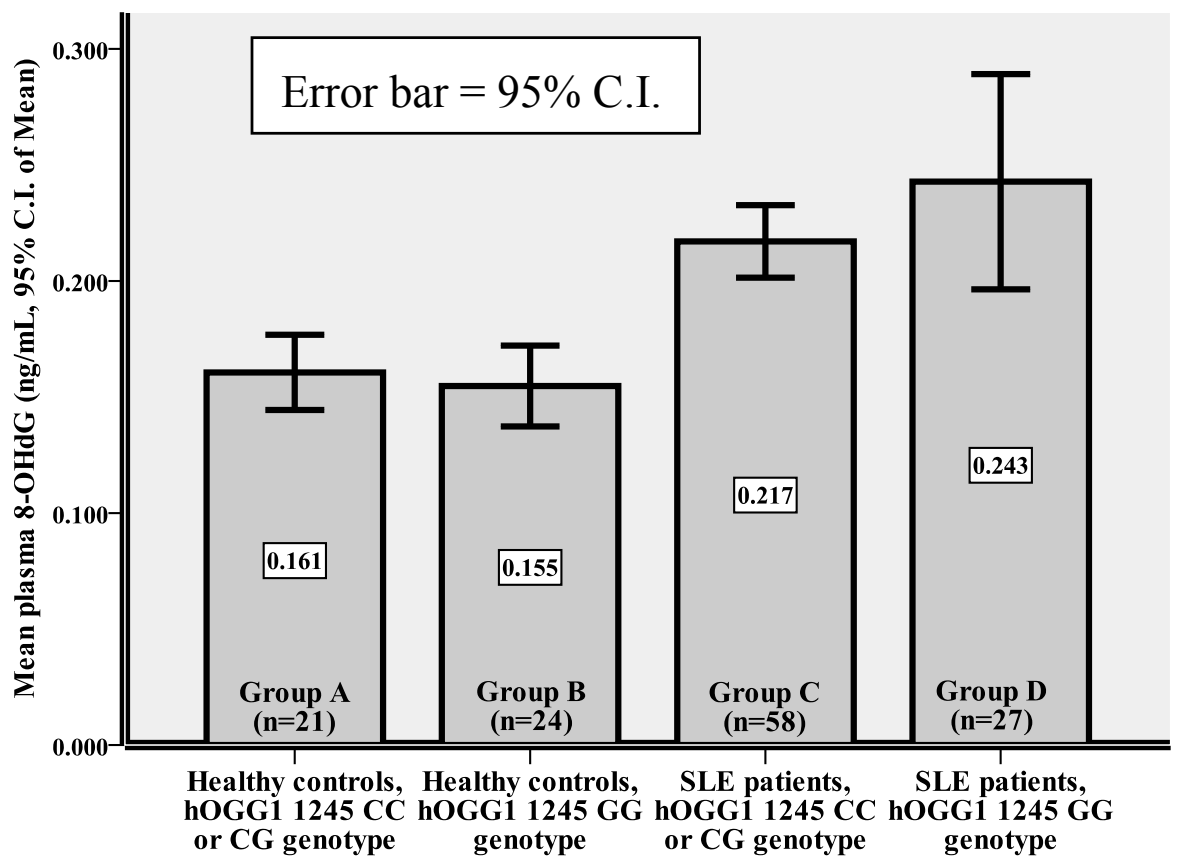

Figure 2. The plasma 8-OHdG in healthy controls and SLE patients with different hOGG1 gene polymorphisms (mean levels and 95\% C.I.).

We have demonstrated that SLE patients had a higher plasma level of 8-OHdG than did the healthy controls [20]. Since 8-OHdG is repaired by hOGG1 in human tissues, the possible influence of $h O G G 1$ 1245 GG genotype on SLE susceptibility or the elevation of 8-OHdG level in SLE patients deserves evaluation. In our study cohort, the percentage of hOGG1 1245 GG genotype in SLE patients was $31.8 \%$, which was lower $(p=0.017)$ than that in healthy controls $(24 / 45,53.3 \%)$. On the contrary, 
the percentage of $h O G G 1 \mathrm{CC}$ genotype in healthy control was $6.7 \%(3 / 45)$, which was lower than in SLE patients $(16.5 \%, 14 / 85)$. As reported in the published data, $h O G G 11245$ GG genotype frequency in SLE patients is variable, higher in normal Taiwanese (20.5\%) population [10] and Japanese populations (13.7\%) [21], similar to normal Chinese population (31.4\%) [18], and lower in another normal Taiwanese cohorts (37.6\% [22] and 42.0\% [19]). There is actually a wide range of frequency in the occurrence of hOGG1 $1245 \mathrm{GG}$ genotype in the normal Taiwanese population. Furthermore, the percentage of CC genotype in our healthy cohort was $6.7 \%$, which was lower than those reported previously $[10,18,19,21,22]$. This might suggest that our healthy cohort had a lower repair activity for oxidative damage to DNA, but it was not that low to lead to a significant elevation of plasma levels of 8-OHdG compared with those found in SLE patients. Our results have indicated that hOGG1 $1245 \mathrm{GG}$ genotype does not predispose to SLE and is not the prominent contributor to a higher plasma 8-OHdG levels in SLE patients. However, if SLE patients harbor the hOGG1 1245 GG genotype (Group D), they might exhibit diminished capacity to ameliorate oxidative stress and resulted in higher plasma levels of 8-OHdG (Table 2 and Figure 2). Alternatively, there might be other contributing factors not yet found that also resulted in higher $8-\mathrm{OHdG}$. Although the $\mathrm{CC}$ genotype was previously reported as a wild type, it was mainly found in the European/American populations. The distribution variations of the hOGG1 gene polymorphisms might be dependent on ethnic group. Thus, the CC, CG or GG genotype cannot be regarded as the wild type of the $h O G G 1$ gene.

\subsection{Distributions of hOGG1 C1245G Polymorphisms in SLE Patients with or without Lupus Nephritis}

As shown in Table 3, the 27 SLE patients who harbored the GG genotype were more susceptible to the development of lupus nephritis compared with the other 58 SLE patients who harbored the CC or CG genotypes $(15 / 27,55.6 \% v s .18 / 58,31.0 \%, p=0.031)$. Thus, for further comparisons, the 85 SLE patients were divided into 4 groups according to the clinical presentations of lupus nephritis and hOGG1 C1245G polymorphism. These include SLE patients without nephritis and with the CC/CG genotypes (Group I), SLE patients without nephritis and with the GG genotype (Group II), SLE patients with nephritis and with the CC/CG genotypes (Group III), and SLE patients with nephritis and with the GG genotype (Group IV).

Table 3. Distribution of $h O G G 1$ gene polymorphisms in SLE patients with or without lupus nephritis.

\begin{tabular}{|c|c|c|c|}
\hline \multirow[b]{2}{*}{ SLE Patients } & \multicolumn{2}{|c|}{ hOGG1 Gene Polymorphisms (Case Number, \%) } & \multirow[b]{2}{*}{$p$-Value } \\
\hline & $\begin{array}{c}\text { hOGG1 } 1245 \text { CC or CG } \\
\text { Genotype }(n=58,100 \%)\end{array}$ & $\begin{array}{c}\text { hOGG1 GG Genotype } \\
(n=27,100 \%)\end{array}$ & \\
\hline Witho & 6)- $-\mathrm{G}_{1}$ & $12(44.4 \%)-\mathrm{Gr}$ & $0.031 *$ \\
\hline With nephritis & $18(31.0 \%)$ - Group 111 & $15(53.6 \%)$ - Uro & \\
\hline \multicolumn{4}{|c|}{$\begin{array}{l}\text { Group I: SLE patients without nephritis and harboring the CC or CG genotypes; Group II: SLE patients } \\
\text { without nephritis and harboring the GG genotype; Group III; SLE patients with nephritis and harboring the } \\
\text { CC or CG genotype; Group IV: SLE patients with nephritis and harboring the GG genotype; * comparison } \\
\text { was made between those harboring CC/CG genotype (Group I + III, } n=58 \text { ) and those harboring GG } \\
\text { genotype (Group II }+ \text { IV, } n=27 \text { ). }\end{array}$} \\
\hline
\end{tabular}




\subsection{Plasma Levels of 8-OHdG in SLE Patients as Related to the Clinical Presentations of Lupus} Nephritis and hOGG1 C1245G Polymorphism

In the patients who harbored the $\mathrm{CC}$ or $\mathrm{CG}$ genotypes (Group I and Group III), there was no significant difference in their plasma levels of $8-\mathrm{OHdG}(0.224 \pm 0.064$ vs. $0.201 \pm 0.046 \mathrm{ng} / \mathrm{mL}$, $p=0.165$ ). In those who harbored the GG genotype (Group II and Group IV), Group IV had higher plasma levels of 8-OHdG than Group II $(0.280 \pm 0.145$ vs. $0.197 \pm 0.040 \mathrm{ng} / \mathrm{mL}, p=0.050)$. In the 33 SLE patients with nephritis (Group III and Group IV), Group IV had higher plasma levels of 8 -OHdG than did Group III $(0.280 \pm 0.145$ vs. $0.201 \pm 0.046 \mathrm{ng} / \mathrm{mL}, p=0.037)$. However, such a difference was not observed between Group I and II in the 52 SLE patients without nephritis $(0.197 \pm 0.040$ vs. $0.224 \pm 0.064 \mathrm{ng} / \mathrm{mL}, p=0.164)$. Furthermore, SLE patients with nephritis who harbored the GG genotype (Group IV) had higher plasma levels of 8-OHdG than SLE patients without nephritis who harbored the CC or CG genotypes (Group I) $(0.280 \pm 0.145$ vs. $0.224 \pm 0.064 \mathrm{ng} / \mathrm{mL}$, $p=0.054)$. Group IV SLE patients with nephritis and with the GG genotype had higher levels of plasma 8-OHdG than did patients in the other groups ( $p=0.020$, ANOVA) (Table 4 and Figure 3 ).

Table 4. Plasma levels of 8-OHdG in SLE patients with or without nephritis as related to the $h O G G 1 \mathrm{C} 1245 \mathrm{G}$ polymorphisms.

\begin{tabular}{|c|c|c|c|c|}
\hline & $\begin{array}{l}\text { Plasma 8-OhdG } \\
(\mathrm{M} \pm \mathrm{SD}, \mathbf{n g} / \mathrm{mL})\end{array}$ & & $\begin{array}{l}\text { Plasma 8-OhdG } \\
(\mathrm{M} \pm \mathrm{SD}, \mathbf{n g} / \mathbf{m L})\end{array}$ & $p$-Value \\
\hline $\begin{array}{l}\text { SLE patients without nephritis } \\
\qquad(n=52)\end{array}$ & $0.218 \pm 0.060$ & $\begin{array}{l}\text { SLE patients with } \\
\text { nephritis }(n=33)\end{array}$ & $0.237 \pm 0.109$ & 0.313 \\
\hline $\begin{array}{c}\text { Group I hOGG1 } 1245 \\
\text { CC or CG genotype }(n=40)\end{array}$ & $0.224 \pm 0.064$ & $\begin{array}{c}\text { Group III } h O G G 11245 \\
\text { CC or CG genotype }(n=18)\end{array}$ & $0.201 \pm 0.046$ & $0.165 *$ \\
\hline $\begin{array}{c}\text { Group II GG genotype } \\
\text { (Cys/Cys } h O G G 1)(n=12)\end{array}$ & $0.197 \pm 0.040$ & $\begin{array}{c}\text { Group IV GG genotype } \\
\text { (Cys/Cys } h O G G 1)(n=15)\end{array}$ & $0.280 \pm 0.145$ & $0.050 *$ \\
\hline \multirow[t]{2}{*}{$p$-value } & $0.164 * *$ & & $0.037 * *$ & $0.054 * * *$ \\
\hline & & & & $0.020 * * * *$ \\
\hline
\end{tabular}

M, mean, SD, standard deviation; * compared between SLE patients without nephritis who harbored the CC or CG genotypes (Group I) and SLE patients with nephritis who harbored the CC or CG genotypes (Group III), and between SLE patients without nephritis who harbored the GG genotype (Group II) and SLE patients with nephritis who harbored the GG genotype (Group IV); ** compared between SLE patients without nephritis who harbored the GG genotype and those harboring the CC or CG genotypes (Group II and Group I), and in SLE patients with nephritis who harbored the GG genotype and those harboring the CC or CG genotype (Group IV and Group III); *** compared between SLE patients with nephritis who harbored the GG genotype and SLE patients without nephritis who harbored the CC or CG genotypes (Group IV and Group I); **** compared among all groups (ANOVA).

It was reported that $h O G G 11245 \mathrm{GG}$ genotype is related to the development of several human degenerative diseases, including type 2 diabetes mellitus [11], Huntington's disease [12], chronic obstructive pulmonary disease [13], chronic renal failure [23] and Graves' ophthalmopathy [14]. Furthermore, the hOGG1 $1245 \mathrm{GG}$ genotype is associated with multiple vessel involvement in patients with coronary artery disease [22], and with the progression of IgA nephropathy [23]. However, the role of $h O G G 1 \mathrm{C} 1245 \mathrm{G}$ polymorphism in lupus nephritis remains unclear. Interestingly, we demonstrated 
that in our SLE cohort, the 27 patients harboring the GG genotype had a significantly higher rate $(15 / 27,55.6 \%)$ of nephritis than the other 58 patients harboring the CC or CG genotypes (18/58, $31.0 \%, p=0.031$, Table 3 ). Several previous studies have shown that the GG genotype is related to the higher 8-OHdG concentration in patients with ESRD $[18,19]$. Thus, it is important to clarify whether the hOGG1 $1245 \mathrm{GG}$ genotype in patients with lupus nephritis confers a higher oxidative damage to DNA compared with SLE patients without nephritis. As shown in Table 4 and Figure 3, SLE patients with nephritis and with the GG genotype (Group IV) had a higher plasma level of 8-OHdG than did other groups of SLE patients ( $p=0.020$, ANOVA). Therefore, we suspect that the SLE patients harboring hOGGl $1245 \mathrm{GG}$ genotype tend to have a higher risk of developing nephritis and accumulating more oxidative damage to DNA in affected tissues.

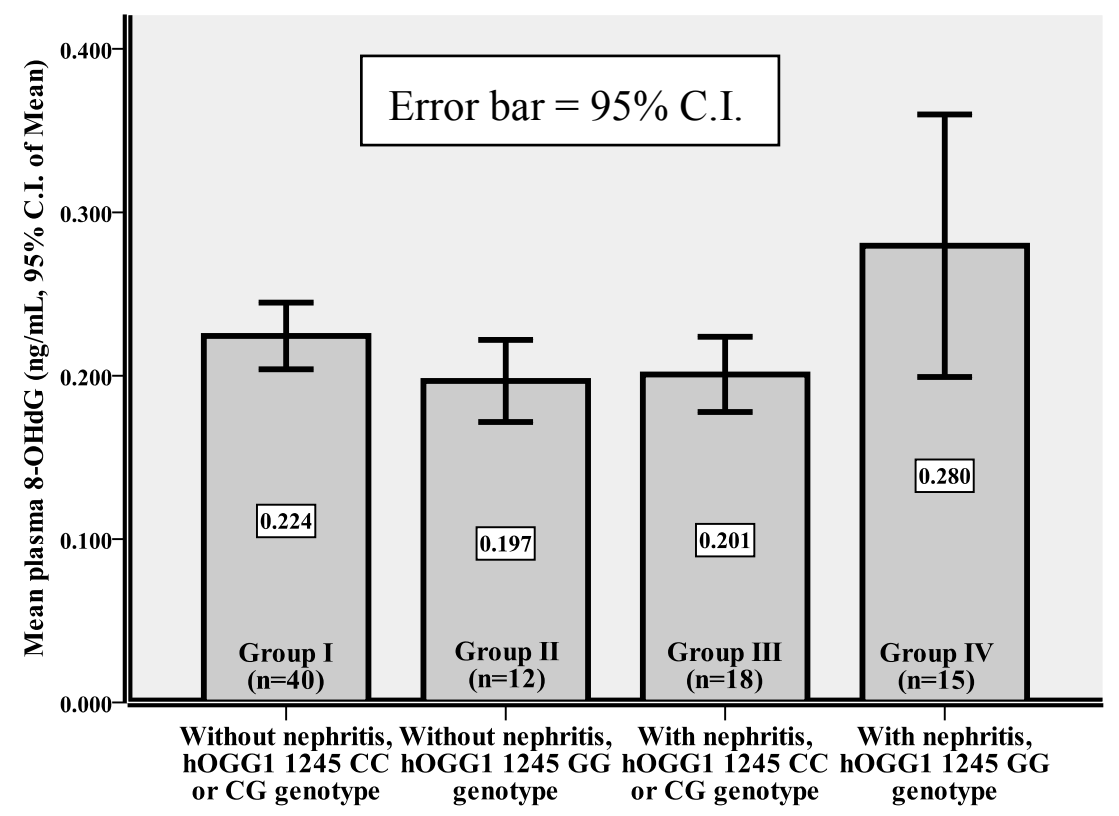

Figure 3. The plasma 8-OHdG in SLE patients with or without nephritis, as related to different $h O G G 1 \mathrm{C} 1245 \mathrm{G}$ genotypes (mean levels and 95\% C.I.).

\section{Experimental Section}

\subsection{Recruitment of SLE Patients and Healthy Controls}

With fulfillment of the American College of Rheumatology (ACR) criteria for the classification of SLE [24], we collected a total of 85 SLE patients (male/female $=12 / 73$ ) with a mean age of 44.6 years from Outpatient Clinic of the Division of Allergy, Immunology and Rheumatology, Taipei Veterans General Hospital. 45 age-matched healthy controls (male/female $=7 / 38$ ) with a mean age of 42.6 years served as the controls. We included the same cohort, their demographic data and plasma levels of 8-OHdG, which had been reported previously for other research purposes [20,25]. Among the 85 SLE patients, $33(38.8 \%)$ were confirmed to have lupus nephritis according to the ACR classification criteria [24,25]. Approval from the Institutional Review Board of Taipei Veterans General Hospital was obtained to conduct this study. 


\subsection{Blood Sample Collection, Plasma Separation and Leukocyte DNA Preparation}

About $10 \mathrm{~mL}$ of venous blood was drawn in a specific tube (VACUETTE ${ }^{\circledR}$, Greiner Bio-one, Monroe, NC, USA) that contained EDTA. After centrifugation at $3000 \times g$ for 10 min at $4{ }^{\circ} \mathrm{C}$, the plasma and leukocyte-enriched buffy coat were collected separately. The plasma was then subject to the determination of $8-\mathrm{OHdG}$. After addition with the erythrocyte (RBC) lysis buffer (Bioman, Taiwan), leukocyte DNA was extracted by standard phenol-chloroform procedures and kept at $-20^{\circ} \mathrm{C}$ until use [26].

\subsection{Determination of 8-OHdG in Plasma by ELISA}

Plasma 8-OHdG was detected by using ELISA kit (High Sensitive 8-OHdG Check ELISA, Japan Institute for the Control of Aging, Nikken SEIL Co., Ltd., Fukuroi, Shizuoka, Japan) according to the manufacturer's instruction [20,27]. Each reaction was done in duplicate and the mean value was used for data presentation.

\subsection{Analysis of hOGG1 C1245G Polymorphisms}

The hOGG1 C1245G polymorphisms were detected by direct genome sequencing. Briefly, $1 \mu \mathrm{L}$ $(10 \mathrm{ng} / \mu \mathrm{L})$ of the leukocyte DNA was amplified in a $50 \mu \mathrm{L}$ PCR reaction that containing $25 \mu \mathrm{L}$ of RBC SensiZyme ${ }^{\circledR}$ Hotstart Taq Premix (RBC Bioscience, Taipei, Taiwan), $22 \mu \mathrm{L}$ of PCR grade $\mathrm{H}_{2} \mathrm{O}, 1 \mu \mathrm{L}$ of each primer (F: 5'-ACTGTCACTAGTCTCACCAG-3' and R: 5'-GGAAGGTGCTTGGGGAAT-3') to generate a 200-bp product [16]. PCR conditions were set as: $95{ }^{\circ} \mathrm{C}$ for $10 \mathrm{~min}$ followed by 40 cycles of amplification at $95{ }^{\circ} \mathrm{C}$ for $30 \mathrm{~s}, 58{ }^{\circ} \mathrm{C}$ for $30 \mathrm{~s}$ and $72{ }^{\circ} \mathrm{C}$ for $60 \mathrm{~s}$, and a final extension at $72{ }^{\circ} \mathrm{C}$ for $7 \mathrm{~min}$. After gel electrophoresis to confirm the DNA band of interest, $30 \mu \mathrm{L}$ of the PCR-amplified product was subjected to direct sequencing (MB Mission Biotech, Taipei, Taiwan). During sequencing, single $\mathrm{C}$ peak at bp1245 indicates a homozygous $\mathrm{CC}$ genotype, single $\mathrm{G}$ peak indicates a homozygous GG genotype, and concurrent $\mathrm{C}$ plus $\mathrm{G}$ peaks indicates the heterozygous $\mathrm{CG}$ genotype $[16,21]$.

\subsection{Statistical Analysis}

The plasma 8-OHdG concentrations are presented as the mean \pm standard deviation $(\mathrm{M} \pm \mathrm{SD})$. The continuous variables between two groups or among three or more groups were compared using the Student's $t$-test, Mann-Whitney $U$-test, ANOVA (analysis of variance) or Kruskal-Wallis test when appropriate. A difference was considered significant when a $p$-value was less than 0.05 .

\section{Conclusions}

In conclusion, we have provided evidence that $h O G G 1 \mathrm{C} 1245 \mathrm{G}$ polymorphism might be one of the multiple factors that confer a higher susceptibility to lupus nephritis and modulate the plasma level of 8-OHdG in patients with SLE. 


\section{Acknowledgments}

This work was partly supported by research grants MOST103-2321-B-715-001 and NSC102-2314B-075-067-MY3 from the Ministry of Science and Technology, Executive Yuan, Taiwan and V103C-10 from Taipei Veterans General Hospital, Taiwan.

\section{Author Contributions}

Hui-Ting Lee, Chen-Sung Lin, Chyou-Shen Lee, Chang-Youh Tsai and Yau-Huei Wei were involved in the study design, preparation and revision of the manuscript and approved the final version for submission. Hui-Ting Lee and Chen-Sung Lin had full access to all of the data during the study and take the responsibility for the integrity and accuracy of the results and data analyses.

\section{Conflicts of Interest}

The authors declare no conflict of interest.

\section{References}

1. Perl, A. Pathogenic mechanisms in systemic lupus erythematosus. Autoimmunity 2010, 43, 1-6.

2. Li, K.J.; Wu, C.H.; Hsieh, S.C.; Lu, M.C.; Tsai, C.Y.; Yu, C.L. Deranged bioenergetics and defective redox capacity in $\mathrm{T}$ lymphocytes and neutrophils are related to cellular dysfunction and increased oxidative stress in patients with active systemic lupus erythematosus. Clin. Dev. Immunol. 2012, 2012, doi:10.1155/2012/548516.

3. Shah, D.; Wanchu, A.; Bhatnagar, A. Interaction between oxidative stress and chemokines: Possible pathogenic role in systemic lupus erythematosus and rheumatoid arthritis. Immunobiology 2011, 216, 1010-1017.

4. Wang, G.; Pierangeli, S.S.; Papalardo, E.; Ansari, G.A.; Khan, M.F. Markers of oxidative and nitrosative stress in systemic lupus erythematosus: Correlation with disease activity. Arthritis Rheumatol. 2010, 62, 2064-2072.

5. Domann, F.E. Aberrant free radical biology is a unifying theme in the etiology and pathogenesis of major human diseases. Int. J. Mol. Sci. 2013, 14, 8491-8495.

6. Neeley, W.L.; Essigmann, J.M. Mechanisms of formation, genotoxicity, and mutation of guanine oxidation products. Chem. Res. Toxicol. 2006, 19, 491-505.

7. Cheng, K.C.; Cahill, D.S.; Kasai, H.; Nishimura, S.; Loeb, L.A. 8-Hydroxyguanine, an abundant form of oxidative DNA damage, causes $\mathrm{G} \rightarrow \mathrm{T}$ and $\mathrm{A} \rightarrow \mathrm{C}$ substitutions. J. Biol. Chem. 1992, 267, 166-172.

8. Boiteux, S.; Radicella, J.P. The human OGG1 gene: Structure, functions, and its implication in the process of carcinogenesis. Arch. Biochem. Biophys. 2000, 377, 1-8.

9. Kohno, T.; Shinmura, K.; Tosaka, M.; Tani, M.; Kim, S.R.; Sugimura, H.; Nohmi, T.; Kasai, H.; Yokota, J. Genetic polymorphisms and alternative splicing of the $h O G G 1$ gene, that is involved in the repair of 8-hydroxyguanine in damaged DNA. Oncogene 1998, 16, 3219-3225. 
10. Chen, S.K.; Hsieh, W.A.; Tsai, M.H.; Chen, C.C.; Hong, A.I.; Wei, Y.H.; Chang, W.P. Age-associated decrease of oxidative repair enzymes, human 8-oxoguanine DNA glycosylases (hOGG1), in human aging. J. Radiat. Res. 2003, 44, 31-35.

11. Sun, C.; Liu, X.; Zhang, H.; Guo, W.; Cai, Z.; Chen, H.; Zhang, K.; Zhu, D.; Wang, Y. Functional polymorphism of $h O G G 1$ gene is associated with type 2 diabetes mellitus in Chinese population. Mol. Cell. Endocrinol. 2010, 325, 128-134.

12. Coppede, F.; Migheli, F.; Ceravolo, R.; Bregant, E.; Rocchi, A.; Petrozzi, L.; Unti, E.; Lonigro, R.; Siciliano, G.; Migliore, L. The hOGG1 Ser326Cys polymorphism and Huntington's disease. Toxicology 2010, 278, 199-203.

13. Yang, S.F.; Xu, Y.J.; Xie, J.G.; Zhang, Z.X. hOGG1 Ser326Cys and XRCC1 Arg399Gln polymorphisms associated with chronic obstructive pulmonary disease. Chin. Med. J. 2009, 122, 960-966.

14. Tanrikulu, S.; Dogru-Abbasoglu, S.; Ozderya, A.; Ademoglu, E.; Karadag, B.; Erbil, Y.; Uysal, M. The 8-oxoguanine DNA $N$-glycosylase 1 (hOGG1) Ser326Cys variant affects the susceptibility to Graves' disease. Cell Biochem. Funct. 2011, 29, 244-248.

15. Park, J.; Chen, L.; Tockman, M.S.; Elahi, A.; Lazarus, P. The human 8-oxoguanine DNA $N$-glycosylase 1 (hOGGl) DNA repair enzyme and its association with lung cancer risk. Pharmacogenetics 2004, 14, 103-109.

16. Lin, C.S.; Wang, L.S.; Chou, T.Y.; Hsu, W.H.; Lin, H.C.; Lee, S.Y.; Lee, M.H.; Chang, S.C.; Wei, Y.H. Cigarette smoking and $h O G G 1$ Ser326Cys polymorphism are associated with 8-OHdG accumulation on mitochondrial DNA in thoracic esophageal squamous cell carcinoma. Ann. Surg. Oncol. 2013, 20, S379-S388.

17. Hassan, S.Z.; Gheita, T.A.; Kenawy, S.A.; Fahim, A.T.; El-Sorougy, I.M.; Abdou, M.S. Oxidative stress in systemic lupus erythematosus and rheumatoid arthritis patients: Relationship to disease manifestations and activity. Int. J. Rheum. Dis. 2011, 14, 325-331.

18. Cai, Z.; Chen, H.; Tao, J.; Guo, W.; Liu, X.; Zheng, B.; Sun, W.; Wang, Y. Association of base excision repair gene polymorphisms with ESRD risk in a Chinese population. Oxid. Med. Cell. Longev. 2012, 2012, 928421.

19. Tarng, D.C.; Tsai, T.J.; Chen, W.T.; Liu, T.Y.; Wei, Y.H. Effect of human OGG1 1245C $\rightarrow$ G gene polymorphism on 8-hydroxy-2'-deoxyguanosine levels of leukocyte DNA among patients undergoing chronic hemodialysis. J. Am. Soc. Nephrol. 2001, 12, 2338-2347.

20. Lee, H.T.; Lin, C.S.; Lee, C.S.; Tsai, C.Y.; Wei, Y.H. Increased 8-hydroxy-2'-deoxyguanosine in plasma and decreased mRNA expression of human 8-oxoguanine DNA glycosylase 1, antioxidant enzymes, mitochondrial biogenesis-related proteins and glycolytic enzymes in leukocytes in patients with systemic lupus erythematosus. Clin. Exp. Immunol. 2014, 176, 66-77.

21. Sugimura, H.; Kohno, T.; Wakai, K.; Nagura, K.; Genka, K.; Igarashi, H.; Morris, B.J.; Baba, S.; Ohno, Y.; Gao, C.; et al. hOGG1 Ser326Cys polymorphism and lung cancer susceptibility. Cancer Epidemiol. Biomark. Prev. 1999, 8, 669-674.

22. Wang, C.L.; Lin, T.H.; Lin, H.Y.; Sheu, S.H.; Yu, M.L.; Hsiao, P.J.; Lin, K.D.; Hsu, C.; Yang, Y.H.; Shin, S.J. The 8-oxoguanine glycosylase 1 (hOGG1) Ser326Cys variant affects the susceptibility to multi-vessel disease in Taiwan coronary artery disease patients. Thromb. Res. 2010, 126, 319-323. 
23. Fukushima, T.; Sasaki, T.; Arakawa, S.; Fujimoto, S.; Horike, H.; Hatta, H.; Kashihara, N. hOGG1 polymorphism correlates with progression of IgA nephropathy. Nephrology 2001, 6, A10-A11.

24. Hochberg, M.C. Updating the American College of Rheumatology revised criteria for the classification of systemic lupus erythematosus. Arthritis Rheumatol. 1997, 40, 1725.

25. Lee, H.T.; Lin, C.S.; Chen, W.S.; Liao, H.T.; Tsai, C.Y.; Wei, Y.H. Leukocyte mitochondrial DNA alteration in systemic lupus erythematosus and its relevance to the susceptibility to lupus nephritis. Int. J. Mol. Sci. 2012, 13, 8853-8868.

26. Liu, C.S.; Kuo, C.L.; Cheng, W.L.; Huang, C.S.; Lee, C.F.; Wei, Y.H. Alteration of the copy number of mitochondrial DNA in leukocytes of patients with hyperlipidemia. Ann. N. Y. Acad. Sci. 2005, 1042, 70-75.

27. Sato, T.; Takeda, H.; Otake, S.; Yokozawa, J.; Nishise, S.; Fujishima, S.; Orii, T.; Fukui, T.; Takano, J.; Sasaki, Y.; et al. Increased plasma levels of 8-hydroxydeoxyguanosine are associated with development of colorectal tumors. J. Clin. Biochem. Nutr. 2010, 47, 59-63.

(C) 2015 by the authors; licensee MDPI, Basel, Switzerland. This article is an open access article distributed under the terms and conditions of the Creative Commons Attribution license (http://creativecommons.org/licenses/by/4.0/). 